\title{
Research article \\ Molecular profiling of breast carcinoma with IHC surrogates in a tertiary care centre in South India
}

\author{
N. Priyathersini ${ }^{1}$, J. Thanka ${ }^{2}$, Jayashree $B^{3}$. \\ ${ }^{1,2,3}$ Department of Pathology, Sri Ramachandra Medical College, Sri Ramachandra Institute of Higher Education and \\ Research, Chennai, India
}

(Received: October $2020 \quad$ Revised: December $2020 \quad$ Accepted: December 2020)

Corresponding author: Jayashree B. Email: jayashreeb0687@gmail.com

\begin{abstract}
Introduction and Aim: Breast cancer is the most common malignancy in females worldwide. Almost 1.4 million new cases have been diagnosed with breast cancer every year. This aims to study the clinicopathological profile and molecular subtypes of invasive breast carcinoma in resected mastectomy specimens over a period of 5 years.

Materials and Methods: A retrospective study of 90 mastectomy and wide local resection specimens received during the period of January 2012 to June 2017 were analyzed. The clinical data of patients including age, gender, and stage of the disease were obtained from the medical records section. Immunohistochemical staining for Estrogen Receptor [ER], Progesterone Receptor [PR] and Human Epidermal Growth Factor Receptor 2 HER2neu were done. The cases were classified according to the molecular classification based on the ER, PR and HER2 receptor status.

Results: The peak incidence of breast carcinoma was in the age group 50 to 60 years. Invasive ductal carcinoma, Not otherwise specified [NOS] accounted for the most common histologic type. There was higher incidence of pT2 tumors in our study. The most common molecular subtype was luminal A, followed by triple negative tumors. These molecular subtypes associated well with Tumor grade and HGDCIS with a statistically significant $p$ value of 0.001 and 0.015 respectively. An increased proportion of Grade 3 tumors were Triple Negative tumors.
\end{abstract}

Conclusion: In breast carcinomas the routine histopathological features provide inexpensive method for understanding tumour biology and prognosis. It's essential in areas with poor resources. ER, PR and HER2 assessment helps in identifying hormonal status and enables for hormone therapy and anti HER2 therapy.

Keywords: Invasive breast carcinoma; Luminal A; Luminal B; triple negative; molecular profiling.

\section{INTRODUCTION}

$\mathrm{B}$ reast cancer is the most common malignancy in females worldwide. Almost 1.4 million new cases have been diagnosed with breast cancer every year. About half of the cases occur in economically developing countries (1). The incidence of breast cancer has grown rapidly during the last decade in many developing countries and accounts for major cause of mortality. The molecular classification of invasive breast carcinoma using global gene profiling is not economical in a poor resource setting. The expression of clinically significant immunohistochemistry (IHC)surrogates has been analysed in many studies. The present study aims at identifying the molecular subtypes using IHC surrogates in our population and analyzing the relation of the molecular subtypes with the other various clinicopathological features of invasive breast carcinoma. The objective was to study the clinicopathological profile and molecular subtypes of invasive breast carcinoma.

\section{MATERIALS AND METHODS}

This is a retrospective study on paraffin blocks of 90 cases of Invasive Breast Carcinoma specimens received in the Department of Pathology at Sri Ramachandra University from 2012 to 2017. Permission of the institutional ethics committee was obtained prior to the commencement of the study (REF: CSP-MED/16/JAN/27/12).

Inclusion criteria: Microscopically proven cases of invasive breast carcinoma of all histological types. Mastectomy and wide local resection specimens received during the period of January 2012 to June 2017.

Exclusion criteria: Breast malignancies other than carcinoma. Trucut core biopsy Specimens

The clinical data of patients including age, gender, and stage were obtained from the medical records section.

The histopathological data were collected from the pathological case files. Paraffin blocks which contained the tumor with adjacent tissue were collected for the study. Five micron sections were cut and stained with hematoxylin and eosin. Tumors were type specified and stage based on WHO guidelines. Invasive breast carcinomas were graded based on the Nottingham combined histologic grade (Elston-Ellis modification of Scarf-Bloom- 
Richardson grading system). Immunostaining for ER was done using monoclonal antibody to estrogen receptor prediluted antibody procured from Biogenex laboratories. Immunostaining for PR was done using Mouse monoclonal antibody to progesterone receptor (Clone: PR88), procured from Biogenex Laboratories. Immunostaining for HER2neu was done using monoclonal antibody to c-erbB-2 Protein
(HER2) prediluted antibody procured from Biogenex Laboratories. ER/PR and HER2 staining were reported as per the American Joint Committee on cancer protocol guidelines. A cutoff of a minimum of $1 \%$ of tumour cells showing nuclear positivity for ER/PR was considered positive (ASCO guidelines, 2010). Reporting Immunohistochemical results of Her2neu was done in the following manner (Table 1).

Table 1: Immunohistochemical results of Her2neu

\begin{tabular}{|c|c|}
\hline IHC Score & Criteria \\
\hline 0 (Negative) & No immunoreactivity or immunoreactivity in < or equal to $10 \%$ of tumour cells. \\
\hline $1+($ Negative $)$ & $\begin{array}{l}\text { Faint weak immunoreactivity in }>10 \% \text { of tumour cells but only a portion of the } \\
\text { membrane is positive. }\end{array}$ \\
\hline $2+($ Equivocal $)$ & $\begin{array}{l}\text { Weak to moderate complete membrane immunoreactivity in }>10 \% \text { of tumour } \\
\text { cells or circumferential intense membrane staining in }<\text { or equal to } 30 \% \text { of cells. }\end{array}$ \\
\hline $3+($ Positive $)$ & $\begin{array}{l}\text { More than } 30 \% \text { of the tumour cells must show circumferential intense and } \\
\text { uniform membrane staining. A homogeneous (chicken wire) pattern should be } \\
\text { present. }\end{array}$ \\
\hline
\end{tabular}

The cases were classified according to the molecular classification based on the ER, PR and HER2 receptor status. (Table 2).

Table 2: Molecular Classification

\begin{tabular}{|c|c|c|c|}
\hline & ER & PR & Her-2/neu \\
\hline Luminal A & + & + & - \\
\hline Luminal B & + & + & + \\
\hline HER2neu & - & - & + \\
\hline Triple negative & - & - & - \\
\hline
\end{tabular}

Statistical analyses: The Data collection was analyzed by using IBM SPSS statistics software 16.0 Version. Categorical variables were tested by using the Pearson's Chi-square test. Statistical significance tested at $\mathrm{p}<0.05$ and data were presented as Number and percentage.

\section{RESULTS}

The study parameters include age, laterality, size of the tumor, clinical staging, histopathological grade, lymph node status and molecular subtyping.

Age: The age of the patients ranged from 20 to 80 years and above. The mean age was 54.5years. Highest incidence was noted in the 51-60year age group accounting for $28 \%$ of the cases. The second highest incidence was seen in the 41-50 years age group accounting for $19 \%$ of cases followed by the age group of 61-70 years (15\%; Graph 1).

Tumor Laterality: The incidence of right breast carcinoma $53 \%$ which is higher when compared to the incidence of left breast carcinoma 37\% (Graph 2). Among the left sided breast carcinoma 19 cases showed nodal positivity, 16 cases showed nodal positivity on the right side (Graph 3).

Histological Subtypes: Among 90 cases studied $83 \%$ of cases were reported as invasive ductal carcinoma. The other histological subtypes were mixed invasive ductal and lobular carcinoma (6\%), Invasive mucinous carcinoma (6\%), invasive papillary carcinoma $(3 \%)$, invasive lobular carcinoma (1\%) and metaplastic carcinoma (1\%) (Graph 4; Fig. 1).

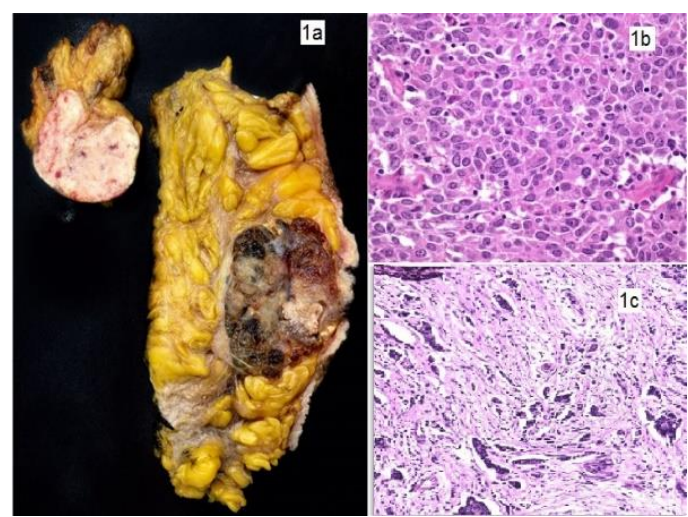

Fig. 1a: Modified Radical Mastectomy specimen showing infiltrating mammary carcinoma. 1b: Infiltrating Ductal carcinoma, NOS Grade 3 H\&E 200x. 1c: Ductolobular carcinoma H\&E 200x 
Histological Grade: Grading of all invasive breast carcinoma was done using Nottingham Histological Grade. The highest incidence was found among grade 2 tumors (48\%), followed by grade $3(28 \%)$. The remaining $14 \%$ were grade 1 (Graph 5 ).

Tumor Stage: Out of 90 cases, $62 \%$ of cases fell under pT2 followed by pT3,pT1 which were $18 \%$ and $12 \%$ respectively. $8 \%$ of cases fell under pT4(Graph 6).

Nodal Status: $49 \%$ of cases presented with N0 status, $19 \%$ with N1 status, $12.2 \%$ with N2 status, $11.1 \%$ with $\mathrm{Nx}$ status and $8.9 \%$ with $\mathrm{N} 3$ status. There was no association between tumor size and lymph node status ( $\mathrm{p}$ value>0.05; Graph 7).

Distant metastasis: All 90 cases fell under M0 category.

\section{High Grade Ductal Carcinoma in Situ (DCIS)}

Out of 90 cases, $46 \%$ of cases had high grade DCIS component. Molecular subtype related well highgrade DCIS ( $p$ value $=0.015$ ). Most of the Luminal A tumors had DCIS component (Graph 8 \& Fig.2).

\section{Molecular Classification}

The most common molecular subtype was Luminal A accounting for $43.3 \%$ of cases, closely followed by Triple Negative subtype with $25.6 \% \%$ of cases. Luminal B and Her 2 subtypes were $16.7 \%$ and $13.3 \%$ respectively (Graph 9 ).

There was statistically insignificant association seen between tumour staging and molecular classification ( $p$ value $=0.054$ ). High proportion of triple negative tumours fell under tumour stage 2 (Graph 11 \& Fig. $3)$.

High grade tumours (Grade 3) are predominantly Triple Negative with a statistically significant association ( $\mathrm{p} \quad$ value $=0.001 ;$ Graph 10).

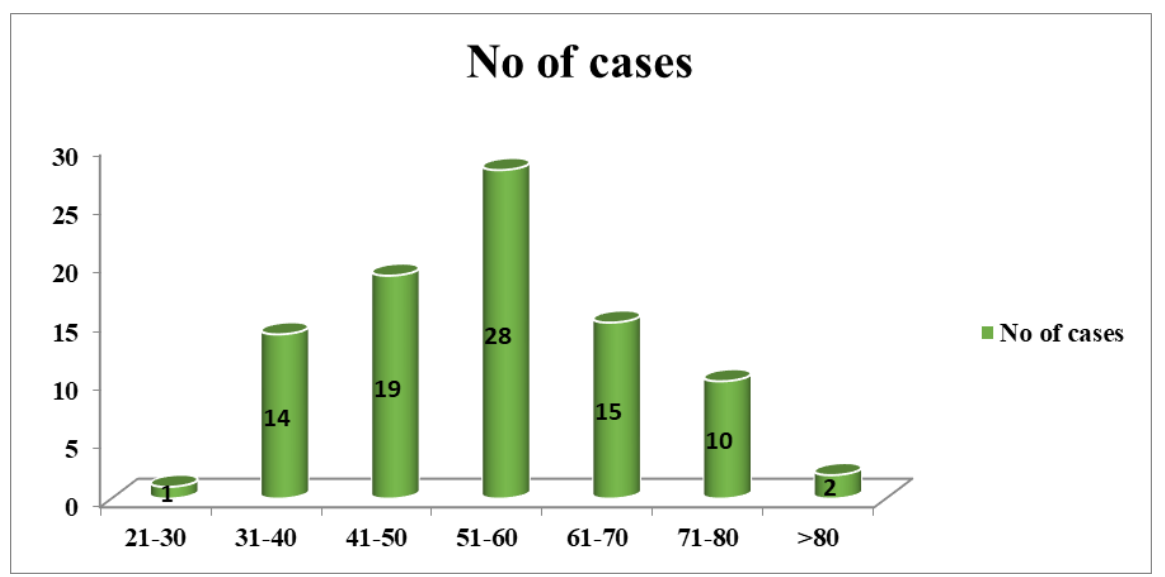

Graph 1: Age wise distribution Age ranged from 21-80 years and above. Maximum number of patients fell in the 51-60 year age group.

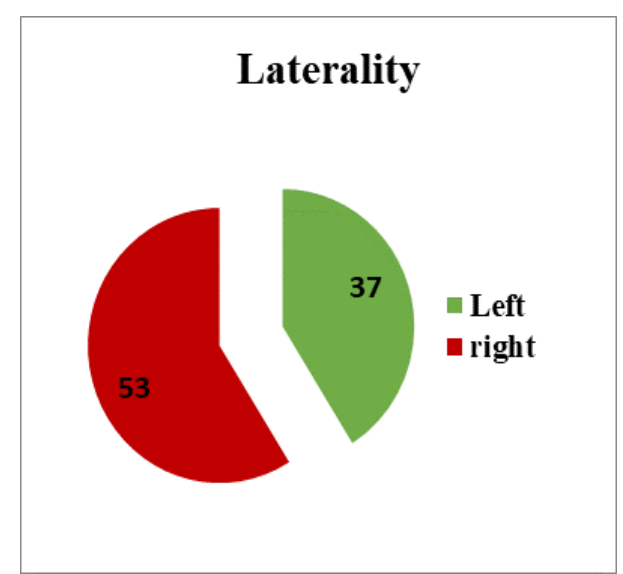

Graph 2: Laterality of the tumor $59 \%$ of the cases were right breast carcinomas. $41 \%$ of cases were left breast carcinomas

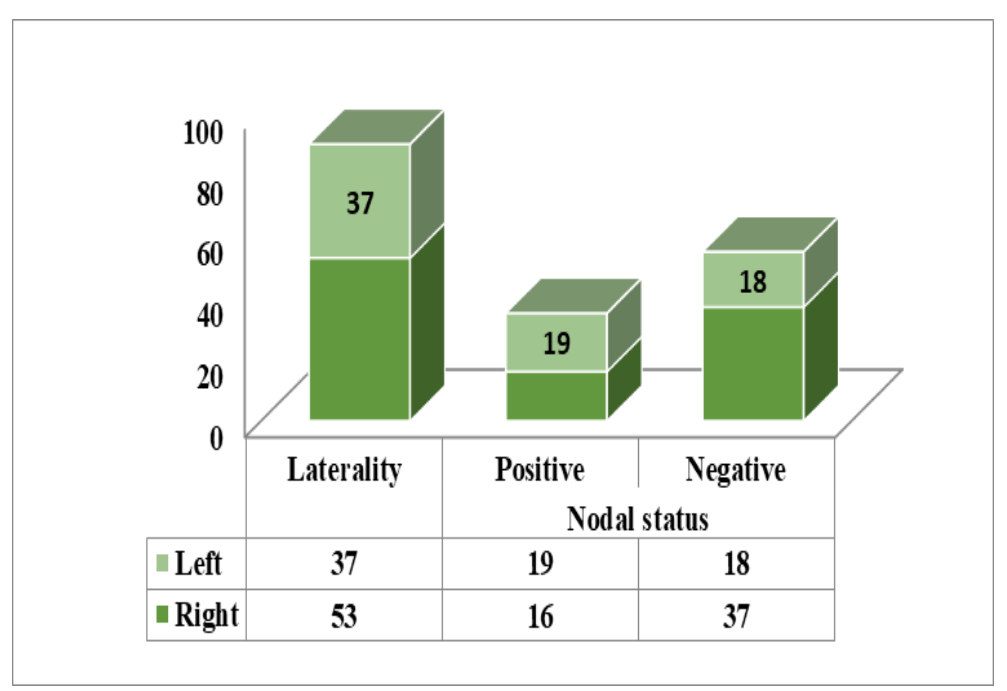

Graph 3: Laterality and Nodal Status

19 nodal positive cases were left sided breast carcinomas and 16 nodal positive cases were right sided breast carcinomas.

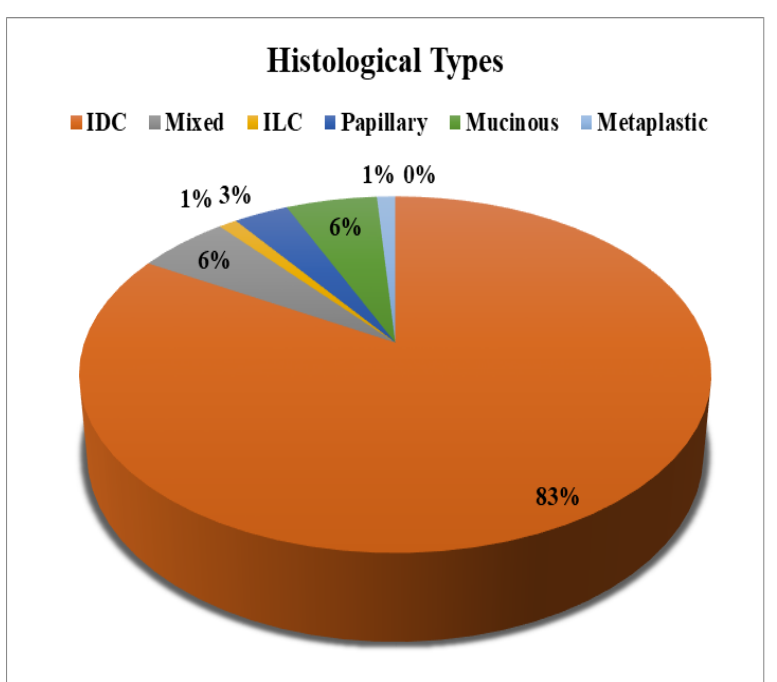

Graph 4: Histological types of invasive breast carcinoma

$83 \%$ of cases were Invasive breast carcinoma, NST 
Priyathersini and Jayashree: Molecular profiling of .... care centre in South India

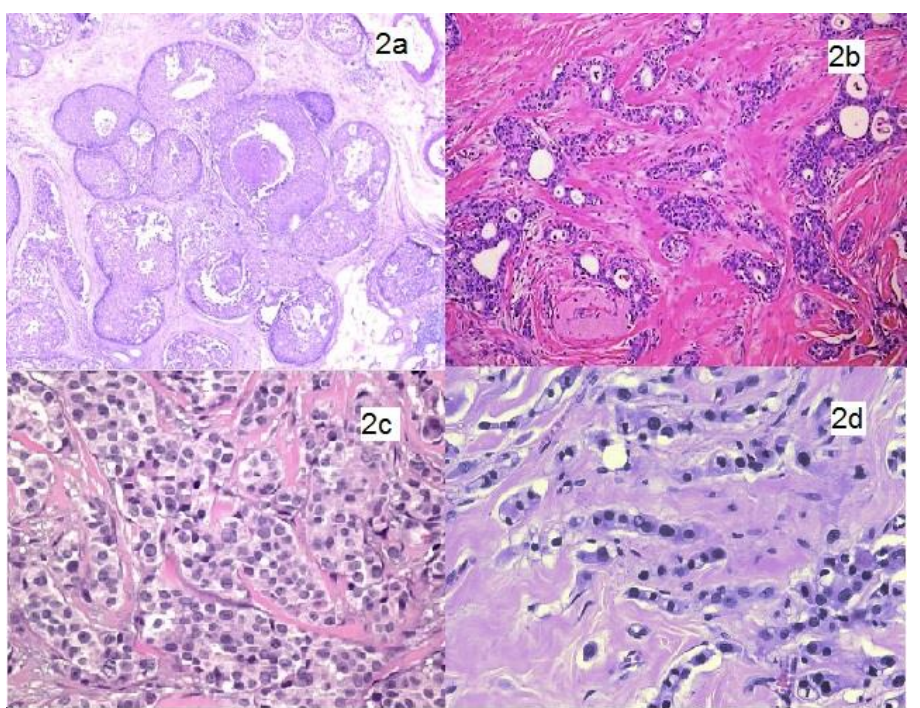

Fig. 2a: High grade Ductal In situ Carcinoma H\&E 100X. Fig.2b: Infiltrating Ductal Carcinoma Grade 1H\&E 200x. Fig.2c: Infiltrating Ductal Carcinoma Grade 2.H\&E 200x.Fig. 2d: Infiltrating Lobular Carcinoma H\&E 200x

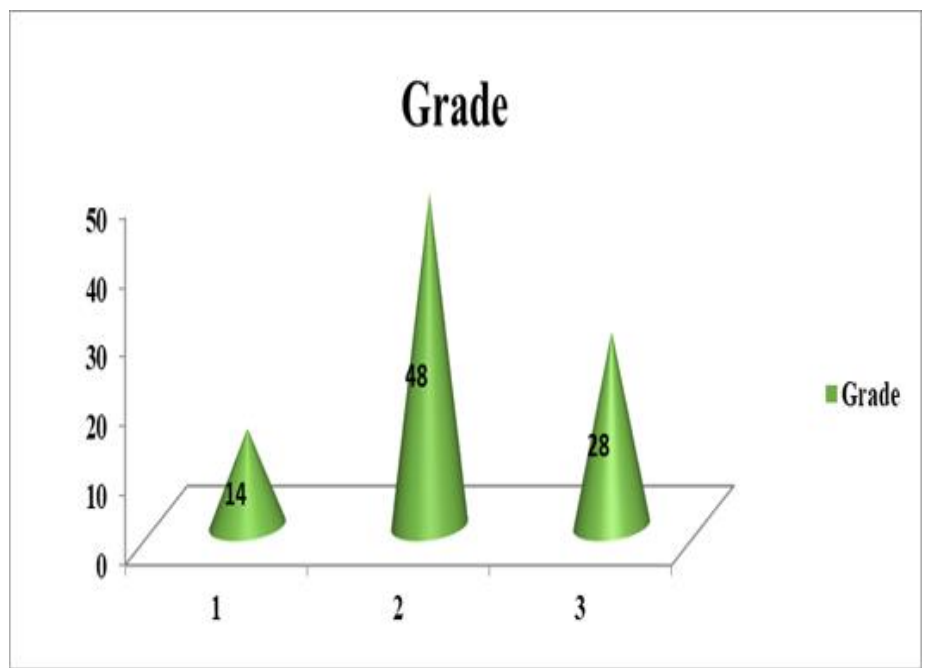

Graph 5: Histological grades of Invasive breast carcinoma $48 \%$ of cases were Grade 2 tumors with the highest incidence

\section{TUMOR STAGE}

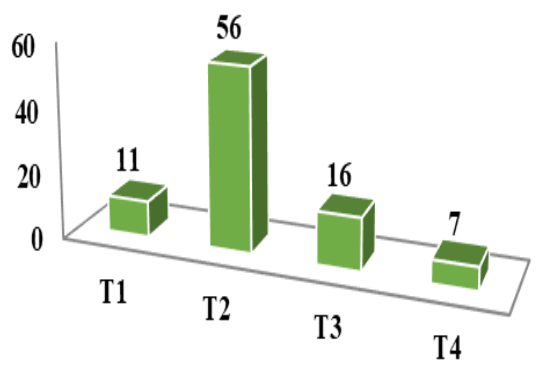

- TUMOR STAGE

Graph 6: Tumor stage (pT). The highest incidence was seen among pT2 tumors accounting for $62 \%$ of cases

\section{NODAL STATUS}

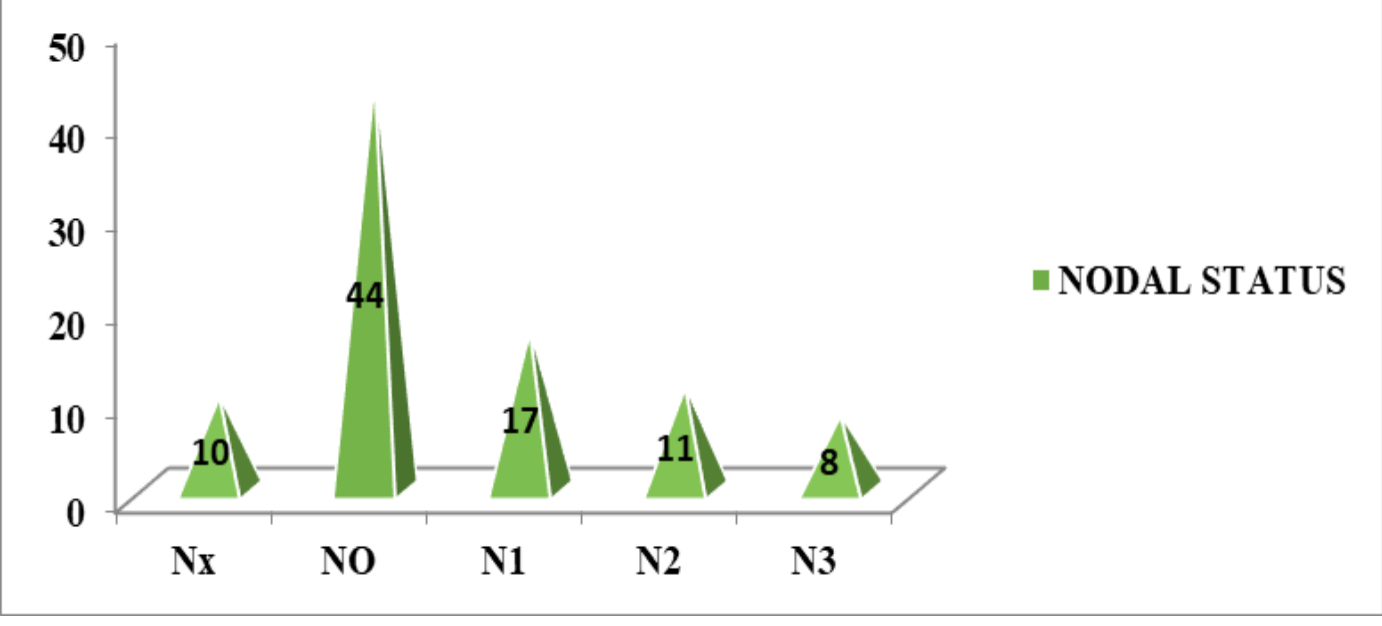

Graph 7: Nodal Status (pN) $49 \%$ of cases presented with pN0 status 

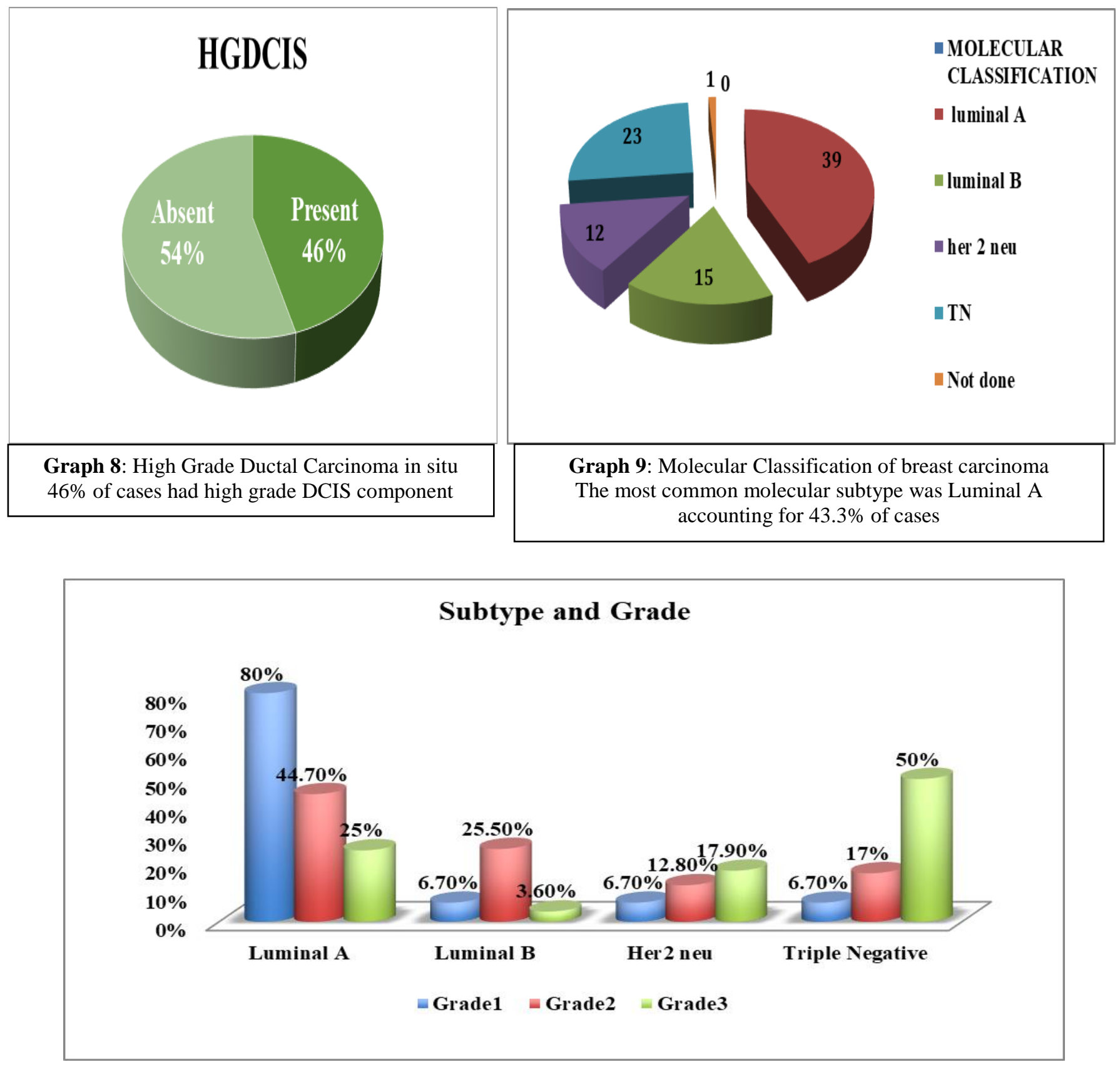

Graph 10: Association between Molecular subtype and Grade

Grade 3 tumors are predominantly triple negative with a statistically significant association ( $\mathrm{p}$ value $=0.001$ ) considered as significant using the Pearson's chi-square test

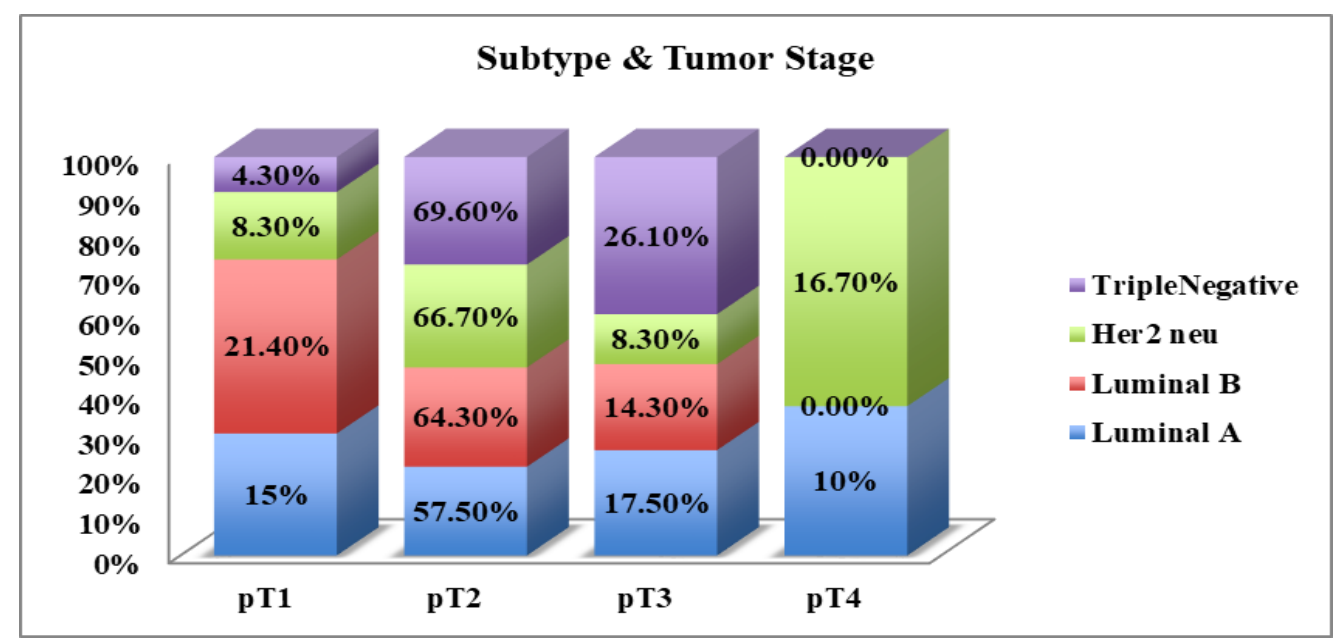

Graph 11: Association of Molecular subtype \& Tumor stage

The statistically insignificant association seen between tumor staging and molecular subtype ( $\mathrm{p}$ value $=0.054$ ) tested by using the Pearson's chi-square test. 


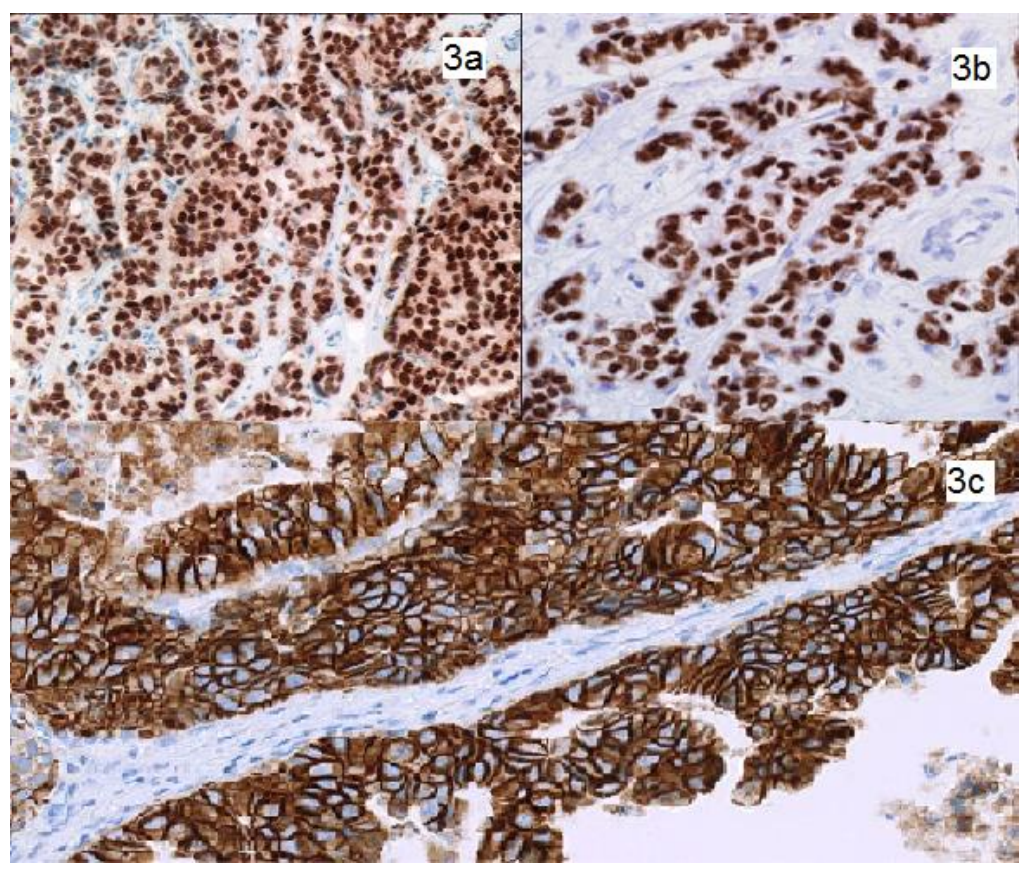

Fig. 3a: ER positive IHC 200x. Fig. 3b: PR positive-IHC 200x

Fig. 3c: Her2 neu positive-IHC 200x

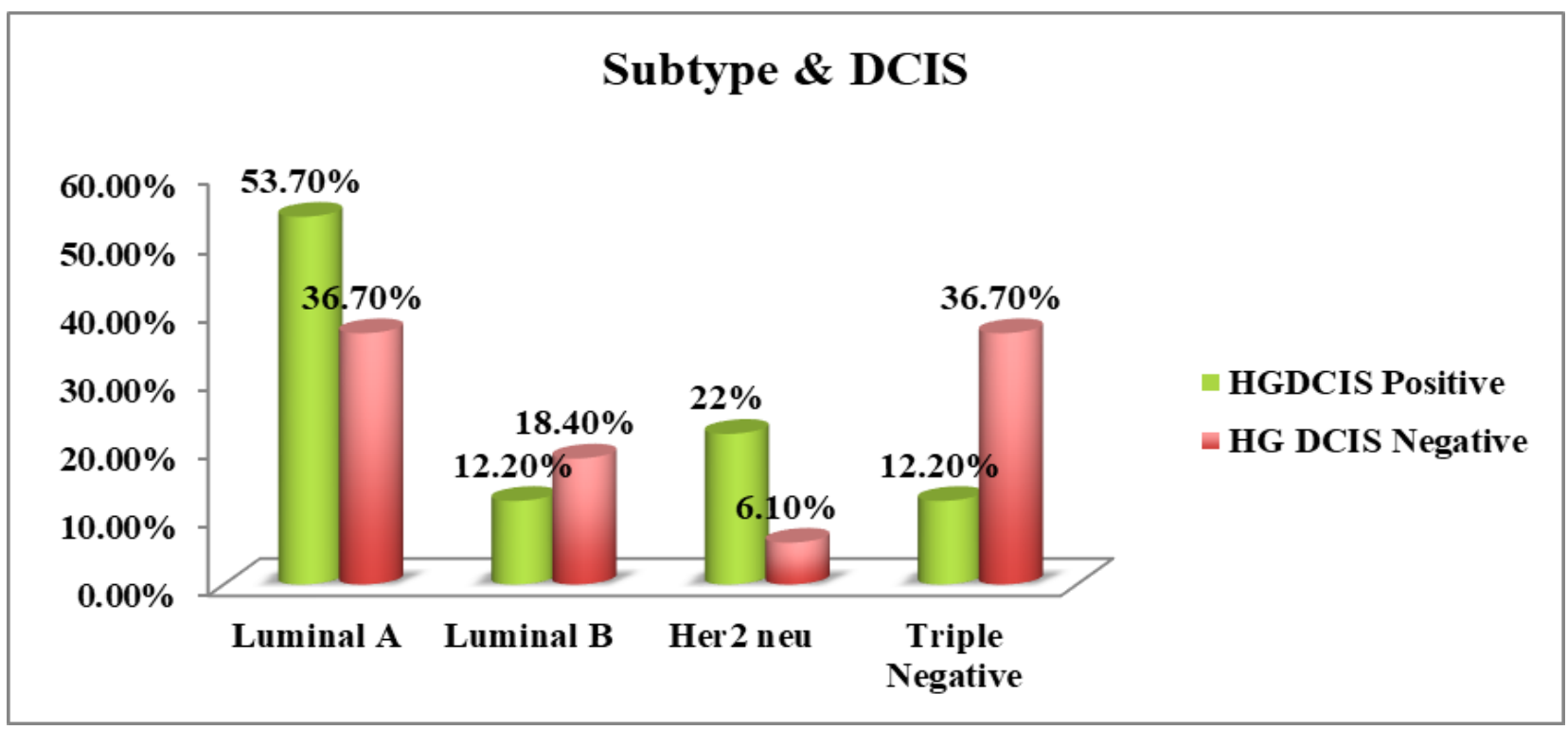

Graph 12: Association of Molecular Subtype \&DCIS

The Statistically significant association seen between Molecular subtype $\&$ highgrade DCIS (p value=0.015) tested by using the Pearson's chi-square test.

\section{DISCUSSION}

The various prognostic factors that determine patient therapy and outcome include age, tumor burden, histological type, grade, lymph node status and hormone receptor status. According to a study done by Leong et al., the peak age for breast cancer is between 40 and 50 years in the Asian countries, whereas the peak age in the Western countries is between 60 and 70 years. In our study, the patient's age ranged from 51-60 years with mean age of 54.5. Studies by Zeeneldin et al., and Weiss et al showed increased preponderance for left breast carcinoma than right $(2,3)$. In a study done by Fatima et al, it was mentioned that right sided breast carcinoma tend to occur at a younger age with higher incidence of nodal metastasis (4). This study showed an increased incidence of right breast carcinoma than left breast carcinoma. Nodal metastasis is seen more in the left breast carcinoma (Graph 3).

Li et al., collected all invasive breast cancer cases for a period of 15 years which showed invasive ductal carcinoma (80.2\%) was the most common type, followed by lobular (11.8\%) and mucinous type $(2.4 \%)$. Saxena et al studied various morphological variants in 569 invasive breast cancers out of which 502 cases $(88.2 \%)$ were invasive ductal carcinoma with no special type (NST). In our study, invasive ductal carcinoma was the most common type 
followed by mixed and papillary carcinoma, mucinous and metaplastic carcinoma in the decreasing order of frequency. Rakha et al., studied around 2,608 cases out of which the author found that Grade 3 tumors (45.6\%) were the highest in the study population (5). A study done in Mumbai, India on 1022 breast cancer patients reported a higher proportion $(70 \%)$ of patients were found to have grade 3 tumours (6). In contrast, we found that the incidence of Grade 2 tumours were high.

According to Leonard et al., patients with high grade ductal carcinoma in situ are at highest risk of developing recurrence ${ }^{(7)}$. Similar results were found in our study with 41 cases of high-grade ductal carcinoma in situ. Breast cancer staging significantly influences the overall survival rate. Diagnosis of breast cancer at an early tumor stage remains vital. According to a study done by Abbas et al., where they studied 60 samples of cases of breast cancer, showed pT2 was the most common group contributing to $56 \%$ (8). Similar results were also seen in our study where $62 \%$ of cases fell under the group pT2. Metastasis to the regional lymph node or internal mammary lymph node has an overall poor survival rate. In our study, 44 cases fell under the category of pN0 status which was seen similar to study by Talvensaari et al., (9).

In our study Luminal A was the most common molecular subtype accounting for $43.3 \%$ of cases, closely followed by triple negative subtype comprising of $25.6 \%$ of cases. Luminal B and Her2 neu comprised of $16.7 \%$ and $13.3 \%$ of cases respectively. Out of the 204 patients with invasive breast carcinoma in a study done by Kim et al., $60.3 \%$ (10). were luminal A, $15.2 \%$ were Triple Negative [TN] which was similar to our study. These molecular subtypes correlated well with tumor grade and high grade ductal carcinoma in situ with a statistically significant $\mathrm{p}$ value of 0.001 and 0.015 respectively. In the same study done by Kim et al., (10), he found significant differences between the subtypes and grade $(\mathrm{p}=0.000)$. Both basal like and Her2 over expressing subtypes were associated with a higher grade, which is also seen in our study.

\section{CONCLUSION}

Breast cancer is heterogeneous neoplasm with many factors affecting its prognosis. The routine histopathological features provide an inexpensive method for understanding the tumor biology and prognosis. It's essential in areas with poor resources. ER, PR and HER2 assessment helps in identifying hormonal status and enables to start the patient on hormone therapy and anti HER2 therapy. The molecular classification can be used an adjunct to the histopathological findings.

The most commonly used IHC surrogates are oestrogen receptor (ER), progesterone receptor (PR), and human epidermal growth factor receptor-2 (HER2), dividing invasive mammary carcinoma into luminal, HER2, and triple-negative subtypes. More genetic features will be available in the future which may throw light into the finer subtypes and ultimately help in accurate prognostication and treatment.

\section{REFERENCES}

1. Sandhu, D. S., Sandhu, S., Karwasra, R. K., Marwah, S. Profile of breast cancer patients at a tertiary care hospital in north India. Indian J Cancer. 2010; 47(1): 16-22.

2. Zeeneldin, A. A., Ramadan, M., Elmashad, N., Fakhr, I., Diaa, A., Mosaad, E. Breast cancer laterality among Egyptian patients and its association with treatments and survival. J Egypt Natl Canc Inst . 2013; 25(4): 199-207.

3. Weiss, H. A., Devesa, S. S., Brinton, L. A. Laterality of breast cancer in the United States. 1996; 7(5): 539-543.

4. Fatima, N., Zaman, M. U., Maqbool, A., Khan, S. H., Riaz, N. Lower incidence but more aggressive behavior of right sided breast cancer in Pakistani women : Does right deserve ore respect? Asian Pac J Cancer Prev. 2013; 14(1): 43-45.

5. Rakha, E. A., Reis-filho, J. S., Baehner, F., Dabbs, D. J., Decker, T., Eusebi, V., et al., Breast cancer prognostic classifi cation in the molecular era : the role of histological grade. Breast Cancer Res. 2010; 12(4): 207.

6. Dinshaw, K. A., Budrukkar, A. N., Chinoy, R. F., Sarin, R., Badwe, R., Hawaldar, R., et al., Profile of prognostic factors in 1022 Indian women with early-stage breast cancer treated with breast-conserving therapy. Int J Radiat Oncol Biol Phys. 2017 ;63(4):1132-1141.

7. Leonard, G. D., Swain, S. M. Ductal carcinoma in situ, complexities and challenges. Am J Roentgenol. 2018; 210(2): 246-255.

8. Soliman, A. A. Histopathological and immunohistochemical study of matrix metalloproteinase-2 and matrix metalloproteinase-9 in breast carcinoma. Journal of the Arab Society for Medical Research. 2017; 12(1): 6-12.

9. Talvensaari-Mattila, A., Pääkkö, P., TurpeenniemiHujanen, T. Matrix metalloproteinase-2 (MMP-2) is associated with survival in breast carcinoma. British journal of cancer. 2003 Oct; 89(7): 1270-1275.

10. Kim, G., Lee, J. S., Choi, Y., Lee, K., Lee, J. H., Nam, J. $\mathrm{H}$., et al., Expression of matrix metalloproteinases and their inhibitors in different immunohistochemical-based molecular subtypes of breast cancer. BMC Cancer. 2014; 14: 959 . 